most important task will now be to match in situ observations of jets with those provided by the International Halley Watch network.

Analysis of mass spectrometry also proved exciting. "When we moved into the coma, we were overwhelmed by the richness of the neutral and ion spectra", said principal investigator D. Krankows$\mathrm{ki}$. The major ions turned out to be those associated with water vapour, $\mathrm{H}_{3} \mathrm{O}^{+}$and $\mathrm{H}_{2} \mathrm{O}^{+}$, with a sharp increase in density to a plateau of about $10^{3}$ per cubic centimetre at about $30,000 \mathrm{~km}$; among the neutrals, $\mathrm{H}_{2} \mathrm{O}$ reached $10^{8} \mathrm{~cm}^{-3}$ at about $3,000 \mathrm{~km}$. The total water outflow was estimated to be about $2 \times 10^{30}$ molecules per second. Compounds of carbon, nitrogen and oxygen were also seen by optical spectrometry. $\mathrm{C}_{2}$ was found to be more abundant than $\mathrm{CN}$, the latter and $\mathrm{CO}^{+}$increasing from a distance of $150,000 \mathrm{~km}$ with a sharp rise in $\mathrm{CO}^{+}$just before approach. Metal ions were also seen, though as $\mathrm{H}$. Balsinger indicated, if the nucleus is a potato it is not a salty one; little sodium was seen.

The blackness of the nucleus may be linked to another surprise reported by Tony McDonnell - the presence of an unexpected population of dust with masses as small as $10^{-17}$ grams. As Vega dust scientist J.S. Simpson remarked, most models predict a fall-off in dust masses below $10^{-12}$ grams, but Vega and Giotto have indicated substantial numbers of smaller particles. It had been thought that such particles would have been expelled from the Solar System by radiation pressure before they could be incorporated into comets. The efficiency of such processes may now have to be re-evaluated in view of the photo-properties of the nucleus discovered by Giotto.

The density of each dust particle seems to have been less than that of water, indicating a porous structure, possibly with a solid core covered with an icy mantle.

Comparing the Giotto and Vega missions, Sagdeev said that the gas, dust and particle measurements showed very good correspondence while highlighting the variability of the neighbourhood of Halley's nucleus. The Giotto measurements of in situ chemistry should be matched with Vega's remote sensing spectrometers, whose resolution might permit investigation even closer to the nucleus than Giotto.

"The science was really great", he said. "After many sleepless nights, we have to part with the comet. We feel real nostalgia now which will last beyond Halley's next apparition."

Meanwhile, Giotto is still working, although with possible damage to the camera and altitude control. It is hoped to adjust its orbit, enabling it to use the Earth as a gravitational sling in 1990 , perhaps to encounter comet Grigg-Skjellerup in 1992.

\title{
Book conservation
}

\section{Red faces over US pilot plant}

\section{Washington}

An explosion and fire have raised design and safety questions about a book conservation process developed by the Library of Congress (LC) that supposedly presented "absolutely no safety risks to personnel or books". The process was designed to make book paper less acidic.

The explosion occurred at a pilot facility operated by Northrop Services Inc., under contract to the National Aeronautics and Space Administration (NASA) at the Goddard Space Flight Center in Greenbelt, Maryland. The facility was designed to support start-up of a $\$ 11.5$ million Mass Deacidification Facility for LC to be constructed at Fort Detrick, Maryland.

What started as a small leak of the chemical used in the deacidication process in early December ultimately resulted in the "controlled disassembly" of the entire system by a demolition team on 21 February.

Book deterioration caused by the high acid content of paper made from wood pulp is a serious problem for all libraries. To decrease the acid content, LC developed and patented a vapour phase process using diethyl zinc (DEZ). The process works by placing books in a vacuum chamber at 20 torr, removing most of the moisture from the books, then injecting gaseous DEZ into the chamber. The DEZ reacts with sulphuric acid in the paper, forming zinc sulphate and water, and the water then reacts with additional DEZ to form zinc oxide in the paper fibres. This provides an alkaline reserve against further acid degradation. Zinc carbonate, produced by adding carbon dioxide to the reaction, also buffers the paper, as well as providing some measure of protection against photodegradation of cellulose.

While the DEZ process has advantages over other liquid processes that have been developed, DEZ is a difficult material to work with. It is pyrophoric in its liquid state at room temperature, burning on contact with air and explosively decomposing on contact with water.

On 5 December, with the book chamber in the pilot facility empty, the system was put through its operational cycle. At the point where all DEZ should have either been recovered or vented, the door to the chamber was allowed to open. The technician on duty reports seeing approximately one litre of liquid DEZ spill out of the chamber onto the ground. Automatic sprinklers extinguished the resulting fire in 8 seconds, and only minor damage resulted, but project engineers immediately shut-down the system while they attempted to discover why liquid DEZ had formed in what was supposed to be a vapour phase of the process. The best guess, according to James Robinson, acting associate director for institutional management at Goddard and chairman of the board of inquiry into the accident, is that some pipes in the gas delivery system had cooled to the point where DEZ would condense, even at low pressure.

When NASA and Northrop engineers returned to the facility early last month, they discovered a positive pressure in the recovery leg of the system. Assuming that additional DEZ or ethane, a reaction byproduct, still remained in some of the process piping, they attempted to vent the remaining gas. During this process, a "significant" explosion occurred, destroying valves, pipes and instrumentation, and compromising the ability to "safe" the system with nitrogen gas.

NASA and Northrop engineers were unable to tell whether any DEZ remained in the process piping, and, if it did, how to remove it safely. Robinson says project engineers considered punching a hole in the remaining pipes from a distance with a rifle bullet, but rejected single-point venting because all remaining DEZ might not escape. NASA and Northrop ultimately concluded that the only way to make the facility safe was to destroy it. An army demolition team set off ten shaped charges on 21 February, "disassembling" the system. The DEZ released at this point burned off in 30 seconds, but this fire spread to the wooden structure housing the system, which burned for nearly an hour. Damage was estimated at $\$ 30,000$.

Both NASA and LC are satisfied that adequate safety procedures were followed in the design of the test facility. If anything, NASA and LC officials say the nature of DEZ prompted excessive safety concerns about its handling.

But safety issues have been raised in the past about how NASA and Northrop conducted the deacidification testing. In 1982, a Northrop safety engineer was fired for insubordination after bypassing his own superiors and reporting safety concerns about the project directly to NASA.

While Robinson would not rule out the possibility that safety issues may have been neglected, he contends "the safety review was adequate... but the fact is that we have had two accidents in three months".

LC has delayed opening of bids for construction of the Mass Deacidification Facility for at least a month until the board of inquiry makes its report.

Peter Sparks, director of preservation for LC, says the pilot system was designed to point out design problems. "We learned a lot from this, but it wasn't quite the way we wanted to learn it."

Joseph Palca 